\title{
Constraining Dark Energy From Splitting Angle Statistic of Strong Gravitational Lenses
}

\author{
Qing-Jun Zhang, Ling-Mei Cheng, and Yue-Liang Wu \\ Kavli Institute for Theoretical Physics China, Institute of Theoretical Physics, \\ Chinese Academy of Science, Beijing 100080, P.R. China \\ ylwu@itp.ac.cn
}

\begin{abstract}
Utilizing the CLASS statistical sample, we investigate the constraint of the splitting angle statistic of strong gravitational lenses(SGL) on the equation-ofstate parameter $w=p / \rho$ of the dark energy in the flat cold dark matter cosmology. Through the comoving number density of dark halos described by PressSchechter theory, dark energy affects the efficiency with which dark-matter concentrations produce strong lensing signals. The constraints on both constant $w$ and time-varying $w(z)=w_{0}+w_{a} z /(1+z)$ from the SGL splitting angle statistic are consistently obtained by adopting a two model combined mechanism of dark halo density profile matched at the mass scale $M_{c}$. Our main observations are: (a) the resulting model parameter $M_{c}$ is found to be $M_{c} \sim 1.4$ for both constant $w$ and time-varying $w(z)$, which is larger than $M_{c} \sim 1$ obtained in literatures; (b) the fitting results for the constant $w$ are found to be $w=-0.89_{-0.26}^{+0.49}$ and $w=-0.94_{-0.16}^{+0.57}$ for the source redshift distributions of the Gaussian models $g\left(z_{s}\right)$ and $g^{c}\left(z_{s}\right)$ respectively, which are consistent with the $\Lambda \mathrm{CDM}$ at $95 \%$ C.L; (c) the time-varying $w(z)$ is found to be for $\sigma_{8}=0.74$ : $\left(M_{c} ; w_{0}, w_{a}\right)=(1.36 ;-0.92,-1.31)$ and $\left(M_{c} ; w_{0}, w_{a}\right)=(1.38 ;-0.89,-1.21)$ for $g\left(z_{s}\right)$ and $g^{c}\left(z_{s}\right)$ respectively, the influence of $\sigma_{8}$ is investigated and found to be sizable for $\sigma_{8}=0.74 \sim 0.90$. After marginalizing the likelihood functions over the cosmological parameters $\left(\Omega_{M}, h, \sigma_{8}\right)$ and the model parameter $M_{c}$, we find that the data of SGL splitting angle statistic lead to the best fit results $\left(w_{0}, w_{a}\right)=\left(-0.88_{-1.03}^{+0.65},-1.55_{-1.88}^{+1.77}\right)$ and $\left(w_{0}, w_{a}\right)=\left(-0.91_{-1.46}^{+0.60},-1.60_{-2.57}^{+1.60}\right)$ for $g\left(z_{s}\right)$ and $g^{c}\left(z_{s}\right)$ respectively.
\end{abstract}

Subject headings: cosmological parameters - cosmology: observations - cosmology: theory - gravitational lensing - quasars: general 


\section{INTRODUCTION}

Since the direct confirmation of the presence of dark energy by Type Ia supernovae (SNe Ia) observation (Riess et.al 1998), the investigation of its property is one of the most important object in cosmology. Many theoretical models have been developed to explain or describe dark energy, which is widely believed as the main component of the cosmological energy today. By now one key to the question seems to be the precise measurement to the equation-of-state parameter $w=p / \rho$ of the dark energy(see a review paper, Peebles \& Ratra 2002). Compared with the data, the limit to the value of parameter $w$ is continuously improved by many experimental groups, including the type Ia supernova(Riess et al. 2004), the Cosmic Microwave Background(CMB)(spergel et al. 2006), and the weak gravitational lenses(WGL)(Weinberg \& Kamionkowski 2002). As a complement to these observations, we shall utilize the splitting angle statistic of strong gravitational lenses(SGL) to quantificationally investigate its constraint on the parameter $w$.

Light lines traversing in the universe are attracted and refracted by the gravitational force of the galaxies on its path, which bring us the signal of the SGL effect, one of which is the multiple images of a single far galaxy. Through comparing the observed number of lenses with the theoretical expected result as a function of image separation and cosmological parameters, it enables us to determine the allowed range of the parameter $w$. Linder (2004) demonstrated that with the addition of strong lensing image separation measurements, the estimates for time-varying $w(z)=w_{0}+w_{a} z /(1+z)$ from CMB, SNe Ia, and WGL could be improved modestly. To see that, we shall carefully in this note investigate the analytic process and the power of SGL data only. There are numerous works studying on the relation between DE and SGL splitting angle statistic (e.g., Porciani \& Madau 2000; Li \& Ostriker 2002; Kuhlen, Keeton \& Madau 2004), but the exact mass density profile of dark halos is unknown yet, which produces the most theoretical uncertainty of the analytic process. The two most widely-used density profiles are the singular isothermal sphere (SIS) profile and the Navarro-Frenk-White (NFW) profile. Some analyses were based solely on the SIS model (e.g., Chae et al. 2002). While from the analyzes by Li \& Ostriker (2002, 2003), and also by Sarbu, Rusin \& Ma (2001), it gave a convictive illumination that a combined mechanism of at least two models can effectively reproduce the observed curve of the lensing probability $P(>\theta)$ to the image splitting angle $\theta$. To achieve this, an additional parameter $\mathrm{M}_{\mathrm{c}}$ is introduced to divide the mass scale of dark halos into different parts as a certain density profile is thought to work only on each part.

The rate of structure growth, which determines the number density of dark halos as the SGL lenses, is very sensitive to the normalization parameter of the matter power spectrum, $\sigma_{8}$. The value of $\sigma_{8}$ is often related to the matter density $\Omega_{M}$ and constrained by the CMB or 
cluster abundance observation. Several years ago, in the $\Lambda$ CDM universe with $\Omega_{M}=0.27$, $\sigma_{8}$ was found to be larger than 0.9 (e.g., Spergel et al. 2003; Wang \& Steinhardt 1998). Then the data of Wilkinson Microwave Anisotropy Probe (WMAP) Three Year (Spergel et al. 2007) provided a noticeably smaller value $\sigma_{8}=0.74 \pm 0.05$ in comparison with the first-year data $\sigma_{8}=0.92 \pm 0.10$. By utilizing the recent clustering results of XMM-Newton soft (0.5-2 keV) X-ray sources, the X-ray clustering, and SNIa data, Basilakos and Plionis (2007) showed that the $\sigma_{8} \approx 0.73$ for $\Omega_{M}=0.26$ and $w=-0.90$, which is consistent with the new result. A smaller $\sigma_{8}$ implies less structure growth at late times and less lensing probability for a single source.

In this paper, by using the CLASS statistical sample (Browne et al.2003), we are going to show how the constraint on the dark energy equation of state parameters $w(z)$ can reliably be obtained for the cases with the assumption of constant $w$ and the time-varying parameterization $w(z)=w_{0}+w_{a} z /(1+z)$, respectively. We highlight three issues which have not previously been investigated. First, we investigate the influences of parameter $w$ on the every step of the lensing process to find the most important point where the change of parameter $w$ shows its effect. Second, by comparing the results for different distributions of sources redshift which have been used in previous works(e.g., Chae et al. 2002; Li \& Ostriker 2002), we illustrate the quantitative influence that is introduced by the uncertainty of the source distribution. Third, we focus on the constraint of the data on the time-varying parameterization $w(z)$. Our paper is organized as follows: Sect. 2 outlines the cosmological model, the mass fluctuations, and the Press-Schechter function used in our calculation. Sect. 3 describes the density profiles and the lensing probabilities. Sect. 4 gives our data analysis and numerical results. The conclusions are presented in the last section.

\section{BASIC CONSIDERATIONS}

In this section we shall describe some ingredients used in our calculations.

\subsection{Cosmological Model and Mass Fluctuations}

During the last two decades more and more observational evidence suggests that our universe at present is accelerated expanding and dominated by a spatially smooth component with negative pressure, so called dark energy. Besides the common cosmological constant supposition, an attractive alternative candidate for dark energy is the potential energy of a slow-varying scalar field, which is conveniently parameterized through $w=p / \rho$. The 
conventional scalar field models, i.e. quintessence models, with a positive kinetic energy term in the field Lagrangian require $w \geq-1$ (e.g., Ratra \& Peebles 1988; Caldwell, Davé, \& Steinhardt 1998), and phantom dark energy , adopting alternatively a negative kinetic energy term, gives the parameter space $w<-1$ (e.g., Caldwell 2002; Carroll, Hoffmann, \& Trodden 2003; Cline, Jeon, \& Moore 2003 ). To fit the data of the observation and give the allowed parameter space, we use two typical parameterizations, i.e., constant $w$ and time-varying $w(z)$, as follows:

$$
\begin{aligned}
& \text { CaseI : } w=\text { constant } \\
& \text { CaseII : } w(z)=w_{0}+w_{a} z /(1+z), \quad w_{a}=d w(z) /\left.d z\right|_{z=0} .
\end{aligned}
$$

The simple form $w(z)=w_{0}+w_{1} z$ is not favored because the value of $w(z)$ runs to infinite when redshift $z$ goes to be infinite. Throughout this paper, we assume a flat cold dark matter universe with present matter density relative to critical density $\Omega_{M}=0.24$ and the Hubble parameter $h=0.73$ (Spergel et al. 2007).

Dark halos of galaxies and galaxy clusters are formed through linear growth and nonlinear collapse of primordial fluctuations of matter in the early universe. The time-varying state-of-equation parameter $w(z)$ is usually realized by models with slow-varying scalar fields, while these scalar fields of dark energy begin to cluster gravitationally and contribute to the perturbation spectrum only at the very large spatial scale $L>100 \mathrm{Mpc}$, which corresponds to a very small wavenumber $k<0.01 \mathrm{Mpc}^{-1}$ (e.g., Ma et al. 1999). In our present considerations, the most concerned length scale $l<1 \mathrm{Mpc}$ is far less than the length scale $L$ and the concerned fluctuations are obtained by integrating the mass power spectrum $k^{2} P(k)$ up to a larger value of the wavenumber $k>1.0 \mathrm{Mpc}^{-1}$. Noticing the fact that the increasing function $k^{2} P(k) \propto k^{3}$ when $k<<1.0 \mathrm{Mpc}^{-1}$, so the contributions from the integral region $k<0.01 \mathrm{Mpc}^{-1}$ are very small, namely the contribution of the dark energy cluster to mass fluctuations can be neglected. Thus we can directly utilize the mass power spectrum of $\Lambda \mathrm{CDM}$ cosmology. In our calculation, the linear CDM power spectrum is computed by adopting the fitting formulae given by (Eisenstein \& Hu 1999)

$$
\Delta_{k}(k, z) \equiv \frac{k^{3}}{2 \pi^{2}} P(k, z)=\delta_{H}^{2}\left(\frac{c k}{H_{0}}\right)^{3+n} T^{2}(k) \mathcal{D}^{2}(z),
$$

The initial power spectrum index $n$ is fixed to be $n \equiv 1$. $T$ is the transfer function

$$
T=\frac{L}{L+C q_{e f f}^{2}},
$$

with

$$
L \equiv \ln \left(e+1.84 q_{e f f}\right), \quad q_{e f f} \equiv \frac{k}{\Omega_{M} h^{2} \mathrm{Mpc}^{-1}}
$$




$$
C \equiv 14.4+\frac{325}{1+60.5 q_{e f f}^{1.11}}
$$

The parameter $\delta_{H}$ is the amplitude of perturbations on the horizon scale today and related to the rms density fluctuations in spheres of radius $r_{8}=8 h^{-1} \mathrm{Mpc}$, so called $\sigma_{8}$ by:

$$
\delta_{H}=\frac{\sigma_{8}}{\left[\int_{0}^{\infty} \frac{d k}{k} \Delta_{k}(k, 0) W^{2}\left(k r_{8}\right)\right]^{1 / 2}}=\frac{\sigma_{8}}{\left[\int_{0}^{\infty} \frac{d k}{k}\left(\frac{c k}{H_{0}}\right)^{3+n} T^{2}(k) W^{2}\left(k r_{8}\right)\right]^{1 / 2}}
$$

where $W(k r)$ is the top-hat window function: $W(k r)=3\left[\frac{\sin (k r)}{(k r)^{3}}-\frac{\cos (k r)}{(k r)^{2}}\right]$. To show the power of SGL data only, we don't use any analytic fitted form of the parameter $\sigma_{8}$, expressed by $\Omega_{M}$ and $w$. Alternatively, unless special clarification we choose to normalize the power spectrum to $\sigma_{8}=0.74$, the best-fit value given by WMAP Three Year data (spergel et al. 2006).

The linear growth function $\mathcal{D}(z)$ is proportional to the linear density perturbation $\delta=$ $\delta \rho_{M} / \rho_{M}$. The evolution of linear perturbation is:

$$
\ddot{\delta}+2 \frac{\dot{a}}{a} \dot{\delta}=4 \pi G \rho_{M} \delta
$$

where $a$ is the scale factor $a=(1+z)^{-1}$, dot means derivative with respect to physical time $t$, the background matter density $\rho_{M}=\rho_{0}(1+z)^{3}, \rho_{0}=\Omega_{M} \rho_{\text {crit }, 0}$ and $\rho_{\text {crit }, 0}=3 H_{0}^{2} /(8 \pi G)$ is today's critical mass density in the universe. Then with the definition $D(z) \equiv \delta(z) / \delta(z=0)$, we can obtain the equation of $\mathcal{D}(a)$ :

$$
\frac{d^{2} \mathcal{D}}{d a^{2}}=\frac{3}{2} \frac{\Omega}{a^{2}} \mathcal{D}-\frac{3}{2 a} \frac{d \mathcal{D}}{d a}[1-w(a)(1-\Omega)]
$$

where $\Omega$ is the matter density parameter $\Omega=\Omega_{M}(1+z)^{3} /\left(H / H_{0}\right)^{2}$ and

$$
\frac{H}{H_{0}}=\frac{\dot{a}}{a H_{0}}=\sqrt{\Omega_{M}(1+z)^{3}+\Omega_{D E} \operatorname{Exp}\left(\int_{0}^{z} d z^{\prime} 3\left[1+w\left(z^{\prime}\right)\right] /\left(1+z^{\prime}\right)\right)}
$$

Here $H_{0}$ is the present Hubble constant $H_{0}=100 h \mathrm{~km} \mathrm{~s}^{-1} \mathrm{Mpc}^{-1}$ and $\Omega_{D E}=\left(1-\Omega_{M}\right)$ for a flat universe. With two boundary conditions: $\left.\mathcal{D}(a)\right|_{a=0}=0$ and $\left.\mathcal{D}(a)\right|_{a=1}=1$, Equation (7) can be calculated out numerically. The second boundary condition means $\mathcal{D}(a)$ is normalized to $\mathcal{D}(a=1)=1$. 


\subsection{Mass Function and Spherical Collapse Approximation}

According to the Press-Schechter theory, the comoving number density of dark halos virialized by redshift $z$ with mass in the range $(M, M+d M)$ is given by

$$
n(M, z) d M=\frac{\rho_{0}}{M} f(M, z) d M .
$$

$f(M, z)$ is the Press-Schechter function. We utilize the modified form by Sheth \& Tormen (1999)

$$
\begin{aligned}
& f(M, z)=-\frac{0.383}{\sqrt{\pi}} \frac{\delta_{c}}{\Delta^{2}} \frac{d \Delta}{d M}\left[1+\left(\frac{\Delta^{2}}{0.707 \delta_{c}^{2}}\right)^{0.3}\right] \times \exp \left[-\frac{0.707}{2}\left(\frac{\delta_{c}}{\Delta}\right)^{2}\right], \\
& \Delta^{2}(M, z)=\int_{0}^{\infty} \frac{d k}{k} \Delta_{k}(k, z) W^{2}(k r)
\end{aligned}
$$

where $\Delta$ is the variance of the fluctuations in a sphere containing a mean mass $M$, and $M$ is related to the length scale $r$ via

$$
M=\frac{4 \pi}{3} r^{3} \rho_{M} .
$$

The parameter $\delta_{c}(z)$ is the linear overdensity threshold for a spherical collapse by redshift $z$. The matter with the overdensity in a certain scale of the early universe would undergo the density growth and the spatial scale reducing. When its average matter density reaches $\delta_{c}(z)$, virialization starts and then a dark halo is formed. In this paper we follow Wang \& Steinhardt (1998) and Weinberg \& Kamionkowski (2003) to calculate the $\delta_{c}(z)$. Under the approximation of spherical tophat collapse and labeling $R$ as the spatial length scale for a halo with a certain mass, the collapse process is determined by the Friedmann equation

$$
\left(\frac{\dot{a}}{a}\right)^{2}=\frac{8 \pi G}{3}\left(\rho_{M}+\rho_{D E}\right)
$$

and the time-time component of the Einstein equations

$$
\frac{\ddot{r}}{r}=-4 \pi G\left[\left(w+\frac{1}{3}\right) \rho_{D E}+\frac{1}{3} \rho_{\text {halo }}\right],
$$

where $\rho_{D E}$ is the energy density of dark energy and $\rho_{\text {halo }}$ is the uniform matter density in the scale $r$. When the scale factor $a(z)$ is very small, i.e., $a(z) \rightarrow 0$ or $z \sim z_{0} \rightarrow \infty$, the equivalent linear overdensity threshold $\delta_{c}(z)$ at $z_{0}$ can approximately be evaluated through the dark halo density $\rho_{\text {halo }}$ and the background matter density $\rho_{M}$, i.e., $\delta_{c}\left(z_{0}\right) \simeq\left(\rho_{\text {halo }} / \rho_{M}-1\right)$. Then utilizing the two boundary conditions: $\left.r(a)\right|_{a=0}=0$ and $d r /\left.d a\right|_{a=a_{t a}}=0$ with $a_{t a}$ the

scale factor at the turn-around time, the function $\delta_{c}(z)$ can be calculated out numerically as follows

$$
\delta_{c}(z)=\delta_{c}\left(z_{0}\right) D(z) / D\left(z_{0}\right), \quad \delta_{c}\left(z_{0}\right) \simeq\left(\rho_{\text {halo }} / \rho_{M}-1\right)
$$


Note that the turn-around time $t_{t a}$ is determined through the virial time $t_{v i r}$ when the overdensity matter starts to form dark halos, i.e., $t_{t a}=t_{v i r} / 2$, which is corresponding to virial redshift $z$ according to the Press-Schechter theory. In this sense, the boundary condition

$d r /\left.d a\right|_{a=a_{t a}}=0$ is related to the virial redshift $z$, so the resulting scale $r(a)$ of dark halos will depend on the virial redshift $z$. As a consequence, the dark halo density $\rho_{\text {halo }}$ actually relies on the virial redshift $z$.

Denote $\Delta_{v i r} \equiv \rho_{\text {halo }} / \rho_{M}$ the ratio of the cluster to the background density. Then the nonlinear overdensity $\Delta_{v i r}(z)$ can also be calculated out directly from the Eq.(11) and Eq.(12) with their two boundary conditions and the $\delta_{c}\left(z_{0}\right)$ given above.

In Fig. 1, we plot the Press-Schechter function $f$ against the mass $M\left(10^{15} h^{-1} M_{\odot}\right)$ of dark halos at redshift $z=0.0,1.5$, and 3.0, respectively. The dash, solid, dot, and dash dot curves are for the typical cases of $w=-0.5, w=-1.0, w=-1.5$, and $w(z)=$ $-1.0-z /(1+z)$. Fig. 2 shows the P-S function $f$ as a function of the redshift $z$ with the dark halo mass $M\left(10^{15} h^{-1} M_{\odot}\right)=0.001,0.1$, and 10 , respectively. Notice the differences of the ordinate length scales. From the two figures, we can see that for larger $M$ and smaller $z$, the P-S function $f$ is more sensitive to the change of the parameter $w$. The explanation is clear: (1) more time passed for the fluctuations to evolve and form dark halos of larger mass, so larger $M$ corresponds to later time; (2) in the early time, the relative proportions of dark energy in the total energy of universe was small and so is its influence on the spatial-time geometry. We can see that even for a small $M \sim 0.001$, the change of $w$ can bring somewhat significant shift of function $f$ in the most range of redshift $z$. This produces the most power of SGL data to constrain the parameter $w$.

\section{DENSITY PROFILE AND LENSING PROBABILITY}

The SGL lensing efficiency is very sensitive to the density profile of dark halos: under the same conditions, the efficiency of SIS model is larger, at least by one order of magnitude, than that of NFW model for the image separation angle $\delta \theta<30^{\prime \prime}$. In this section, we shall discuss the influence of different $w$ on the lensing probability $P\left(>\Delta \theta_{0}\right)$ for SIS profile and NFW profile, respectively. 


\subsection{SIS Profile as a Lens}

The SIS profile has a simple spherically symmetric form (Schneider, Ehlers, \& Falco 1992)

$$
\rho(r)=\frac{\sigma_{v}^{2}}{2 \pi G} \frac{1}{r^{2}}
$$

where $\sigma_{v}$ is the velocity dispersion, which can be related to the mass $M$ of a dark halo via $\sigma_{\nu}^{2}=G M / 2 r_{v i r}$ after integrating the density function $\rho(r)$ from $r=0$ to $r=r_{v i r}$. Here $r_{v i r}$ is the viral radius of a dark halo, which is commonly defined by demanding that the mean density within the virial radius of the halo be a factor $\Delta_{v i r}$ times larger than the background density, $\rho_{M}$, i.e. $M=\frac{4 \pi}{3} \Delta_{v i r} \rho_{c r} r_{v i r}^{3}$. Eliminating the dependance on the $r_{v i r}$, we get

$$
M=\frac{\sigma_{v}^{3}}{G}\left(\frac{6}{\pi \Delta_{v i r} G \rho_{c r}}\right)^{1 / 2} .
$$

This profile is supported by the observed flat rotation curves of the spiral galaxies and is widely utilized in the gravitational lensing anslysis. Due to its symmetry, the lensing analysis is quite easy. Integrate the density component along the line of sight and then we get its surface mass density

$$
\Sigma(\xi)=\frac{\sigma_{v}^{2}}{2 G} \frac{1}{\xi}
$$

where $\xi \equiv|\boldsymbol{\xi}|$ and $\boldsymbol{\xi}$ is the position vector in the lens plane. The lensing equation is given by

$$
\boldsymbol{\eta}=\frac{D_{S}^{A}}{D_{L}^{A}} \boldsymbol{\xi}-D_{L S}^{A} \boldsymbol{\alpha}(\boldsymbol{\xi}),
$$

where $\boldsymbol{\eta}$ is the source position. $D_{S}^{A}, D_{L}^{A}$, and $D_{L S}^{A}$ are the angular-diameter distances from the observer to the source, from the observer to the lens and from the lens to the source. The angle $\boldsymbol{\alpha}(\boldsymbol{\xi})$ is the gravitational deflection angle. For a circularly-symmetric surface mass density, $\Sigma(\boldsymbol{\xi})=\Sigma(\xi)$, images appear on the plane defined by the observer point, the lens center, and the source position, and the angle $\alpha(\xi)$ is given by

$$
\alpha(\xi)=\frac{8 \pi G}{c^{2} \xi} \int_{0}^{\xi} \xi^{\prime} \Sigma\left(\xi^{\prime}\right) d \xi^{\prime}
$$

To simplify the lensing equation, we define the length scales in the lens plane and the source plane as

$$
\xi_{0}=4 \pi\left(\frac{\sigma_{v}}{c}\right)^{2} \frac{D_{L}^{A} D_{L S}^{A}}{D_{S}^{A}}, \quad \eta_{0}=\xi_{0} \frac{D_{S}^{A}}{D_{L}^{A}}
$$


Then the position vector of a point in the lens plane or source plane is $\boldsymbol{\xi}=\mathbf{x} \xi_{0}$ or $\boldsymbol{\eta}=\mathbf{y} \eta_{0}$. After the reduction, the lensing equation for an SIS lens is given by

$$
y=x-\frac{|x|}{x} .
$$

It is easy to see that when $|y| \leq 1$, i.e. $|x| \leq 1$, a single source has double images with the separation $\Delta x \equiv 2$ and the splitting angle

$$
\Delta \theta=\frac{\xi_{0}}{D_{L}^{A}} \Delta x=8 \pi\left(\frac{\sigma_{v}}{c}\right)^{2} \frac{D_{L S}^{A}}{D_{S}^{A}}=\frac{8 \pi G}{c^{2}} \frac{D_{L S}^{A}}{D_{S}^{A}}\left(\frac{\pi M^{2} \rho_{M} \Delta_{v i r}}{6}\right)^{1 / 3}
$$

Hence the cross section for two images with a splitting angle $\Delta \theta>\Delta \theta_{0}$ is given by

$$
\sigma=\pi \xi_{0}^{2} \vartheta\left(\Delta \theta-\Delta \theta_{0}\right)=\pi \xi_{0}^{2} \vartheta\left(M-M_{0}\right)
$$

where $\vartheta$ is the step function, and $\mathrm{M}_{0}$ related with $\Delta \theta_{0}$ can be solved from the equation(20)

The probability for a source at redshift $z_{s}$ undergoing a lensing event on account of the galaxies distribution from the source to the observer can be obtained by dividing the total lensing cross-section by the area $A(z)$ of the lens plane, (Schneider et al. 1992)

$$
P=\int_{0}^{z_{s}} \int_{0}^{\infty} \frac{d D_{L}}{d z}(1+z)^{3} n(M, z) \sigma(M, z) d M d z,
$$

where $D_{L}$ is the proper distance from the observer to the lens.

Inserting equation (8) and (21) into equation (22), we have for the SIS case (Li \& Ostriker 2002)

$$
\begin{aligned}
\frac{d P\left(>\Delta \theta_{0}\right)}{d z}=16 & \pi^{3} \rho_{\text {crit }, 0} \Omega_{M}(1+z)^{3} \frac{d D_{L}}{d z}\left(\frac{D_{L}^{A} D_{L S}^{A}}{D_{S}^{A}}\right)^{2} \\
& \times \int_{0}^{\infty} \frac{f(M, z)}{M}\left(\frac{\sigma_{v}}{c}\right)^{4} \vartheta\left(M-M_{0}\right) d M .
\end{aligned}
$$

Differentiate this expression with respect to $\Delta \theta_{0}$, we can obtain the probability density for a source to have a double image with splitting angle $\Delta \theta=\Delta \theta_{0}$ :

$$
\begin{aligned}
\frac{d^{2} P\left(>\Delta \theta_{0}\right)}{d \Delta \theta_{0} d z} & =16 \pi^{3} \rho_{\text {crit }, 0} \Omega_{M}(1+z)^{3} \frac{d D_{L}}{d z}\left(\frac{D_{L}^{A} D_{L S}^{A}}{D_{S}^{A}}\right)^{2} \frac{f\left(M_{0}, z\right)}{M_{0}}\left(\frac{\sigma_{v}\left(M_{0}\right)}{c}\right)^{4} \frac{d M_{0}}{d \Delta \theta_{0}} \\
\frac{d M_{0}}{d \Delta \theta_{0}} & =\frac{3 c^{2}}{16 \pi G}\left(\frac{\pi \rho_{M} \Delta_{v i r}}{6}\right)^{2 / 3} \frac{D_{S}^{A}}{D_{L S}^{A}} M_{0}^{1 / 3} .
\end{aligned}
$$

The proper distance $D^{L}$ and the angular-diameter distance $D^{A}$ from the redshift $z_{1}$ to $z_{2}$ are calculated via

$$
D^{L}\left(z_{1}, z_{2}\right)=\int_{z_{1}}^{z_{2}} \frac{d z}{(1+z) H(z)}, \quad D^{A}\left(z_{1}, z_{2}\right)=\frac{1}{1+z_{2}} \int_{z_{1}}^{z_{2}} \frac{d z}{H(z)} .
$$




\subsection{NFW Profile as a Lens}

The NFW profile, based on the N-body numerical simulation of cold dark matter, is a very important approach for understanding the formation of galaxies and clusters of galaxies (e.g., Zhao 1996; Hanyu \& Habe 2001). Its mass density is given by (Navarro, Frenk \& White, 1995, 1996, 1997)

$$
\rho_{\mathrm{NFW}}(r)=\frac{\rho_{s} r_{s}^{3}}{r\left(r+r_{s}\right)^{2}},
$$

where $\rho_{s}$ and $r_{s}$ are constants:

$$
\rho_{s}=\frac{\rho_{M} \Delta_{v i r}}{3} \frac{c_{1}^{3}}{f\left(c_{1}\right)}, \quad r_{s}=\frac{1}{c_{1}}\left(\frac{3 M}{4 \pi \rho_{M} \Delta_{v i r}}\right)^{1 / 3},
$$

with $f\left(c_{1}\right)=\ln \left(1+c_{1}\right)-c_{1} /\left(1+c_{1}\right)$. For the concentration parameter $c_{1}$, we adopt the fitting formulae given by Bullock et al. $(2001): c_{1}=9(1+z)^{-1}\left(\mathrm{M} / 1.5 \times 10^{13} h^{-1} \mathrm{M}_{\odot}\right)^{-0.13}$.

Similar to the case of SIS, we define the position vector in the lens plane and the source plane as $\boldsymbol{\xi}=\mathbf{x} r_{s}$ and $\boldsymbol{\eta}=\mathbf{y} r_{s} D_{S}^{A} / D_{L}^{A}$, respectively. The surface mass density for the NFW profile is given by(Li \& Ostriker 2002)

$$
\Sigma(x)=2 \rho_{s} r_{s} \int_{0}^{\infty}\left(x^{2}+z^{2}\right)^{-1 / 2}\left[\left(x^{2}+z^{2}\right)^{1 / 2}+1\right]^{-2} d z
$$

Then the reduced lensing equation is

$$
y=x-\mu_{s} \frac{s(x)}{x},
$$

where

$$
\begin{aligned}
& \mu_{s} \equiv 4 \rho_{s} r_{s} / \Sigma_{\mathrm{cr}} \\
& \Sigma_{\mathrm{cr}} \equiv \frac{1}{4 \pi G} \frac{D_{S}^{A}}{D_{L}^{A} D_{L S}^{A}}, \\
& s(x) \equiv \int_{0}^{x} u d u \int_{0}^{\infty}\left(u^{2}+z^{2}\right)^{-1 / 2}\left[\left(u^{2}+z^{2}\right)^{1 / 2}+1\right]^{-2} d z,
\end{aligned}
$$

The dimensionless parameter $\mu_{s}$ determines the size of the lensing cross-section $\sigma$ for a NFW halo to produce multiple images: larger $\mu_{s}$, smaller $\sigma$. The curve of y to x runs through the coordinate origin and has a extremum point central-symmetrically on each side, whose coordinates $\left(x_{c r}, y_{c r}\right)$ are determined by $d y /\left.d x\right|_{x_{c r}}=0$ and $y_{c r}=y\left(x_{c r}\right)$. Thus a single source with a certain $y$ has multiple images when $|y| \leq y_{\mathrm{cr}}$. Once more than two images are formed, 
we shall only consider the splitting angle $\Delta \theta$ between the two outside images. According to Li \& Ostriker 2002, we shall neglect the variety of $\Delta \theta$ caused only by the movement of y and get $\Delta x(y) \approx \Delta x(y=0)=2 x_{0}$, where $x_{0}$ is the positive root of $y(x)=0$. Then the splitting angle $\Delta \theta$ is given by

$$
\Delta \theta=\frac{r_{s}}{D_{L}^{A}} \Delta x \approx \frac{2 x_{0} r_{s}}{D_{L}^{A}} .
$$

and the cross-section for forming multiple images with $\Delta \theta>\Delta \theta_{0}$ is

$$
\sigma\left(>\Delta \theta_{0}, M, z\right) \approx \pi y_{\mathrm{cr}}^{2} r_{s}^{2} \vartheta\left(\Delta \theta-\Delta \theta_{0}\right)
$$

Fig. 3 shows the splitting angle $\Delta \theta$ as the function of $M\left(10^{15} h^{-1} M_{\odot}\right)$ in SIS and NFW cases for $w=-0.5, w=-1.0, w=-1.5$, and $w(z)=-1.0-z /(1+z)$. The source object is at $z_{s}=1.5$, and the lens object is at $z=0.3$. In Fig. 4 we plot the $\Delta \theta$ against the redshift of lens $z$ for $M=0.01,1.0$, and 100 , respectively. The source object is at $z_{s}=1.5$. The curves of $w(z)$ and $w=-1.0$ almost overlap in both figures. We can see that the $\Delta \theta$ produced by a NFW lens is more sensitive to the parameter $w$ than that of an SIS lens, especially for small $M$. For SIS case, There are only quite small changes of $\Delta \theta$ for our different selections of $w$ in both Fig. 3 and Fig. 4.

Fig. 5 gives the cross section $\sigma$ against the redshift of lens $z$ for $M\left(10^{15} h^{-1} M_{\odot}\right)=0.01$, 1.0, and 100, respectively. The source object is at $z_{s}=1.5$. For SIS case, There are not visible changes of $\Delta \theta$ for our different selections of $w$. For NFW case, the cross section is more sensitive to the parameter $w$ for smaller $M$ and somewhat smaller $z$.

Using equations (8),$(22)$, and (32), we then get the differential lensing probability for the NFW case (Li \& Ostriker 2002)

$$
\frac{d P\left(>\Delta \theta_{0}\right)}{d z}=\pi \rho_{\text {crit }, 0} \Omega_{M}(1+z)^{3} \frac{d D_{L}}{d z} \int_{0}^{\infty} \frac{f(M, z)}{M} y_{\mathrm{cr}}^{2} r_{s}^{2} \vartheta\left(M-M_{0}\right) d M .
$$

Differentiate this expression with respect to $\Delta \theta_{0}$, we can obtain the probability density for a source to have a double image with splitting angle $\Delta \theta=\Delta \theta_{0}$ :

$$
\begin{aligned}
\frac{d^{2} P\left(>\Delta \theta_{0}\right)}{d \Delta \theta_{0} d z} & =\pi \rho_{c r i t, 0} \Omega_{M}(1+z)^{3} \frac{d D_{L}}{d z} \frac{f\left(M_{0}, z\right)}{M_{0}} y_{\mathrm{cr}}^{2}\left(M_{0}\right) r_{s}^{2}\left(M_{0}\right) \frac{d M_{0}}{d \Delta \theta_{0}} \\
\frac{d M_{0}}{d \Delta \theta_{0}} & =\frac{D_{L}^{A}}{2}\left(r_{s}\left(M_{0}\right) \frac{d x_{0}}{d M_{0}}+x_{0}\left(M_{0}\right) r_{s}\left(M_{0}\right) \frac{1 / 3+0.13}{M_{0}}\right)^{-1} \\
\frac{d x_{0}}{d M_{0}} & =\frac{g\left(x_{0}\right) / x_{0} d \mu_{s} / d M_{0}}{1-\mu_{s}\left(M_{0}\right)\left(g^{\prime}\left(x_{0}\right) / x_{0}-g\left(x_{0}\right) / x_{0}^{2}\right)} \\
\frac{d \mu_{s}}{d M_{0}} & =\frac{4 r_{s} \rho_{s}}{M_{0} \Sigma_{c r}}\left(0.07+\frac{0.13 c_{1}^{2}}{\left(1+c_{1}\right)^{2} f\left(c_{1}\right)}\right)
\end{aligned}
$$




$$
g^{\prime}\left(x_{0}\right)=x_{0} \int_{0}^{\infty}\left(x_{0}^{2}+z^{2}\right)^{-1 / 2}\left[\left(x_{0}^{2}+z^{2}\right)^{1 / 2}+1\right]^{-2} d z
$$

\section{DATA ANALYSIS AND NUMERICAL RESULTS}

The CLASS statistical sample provided a well-defined statistical sample with $N=8958$ sources. Totally $N_{l}=13$ multiple image gravitational lenses have been discovered and all have image separations $\Delta \theta<3^{\prime \prime}$ (Browne et al. 2003). The data informations of the 13 observed lens systems are not entire: the source redshift $z_{s}$ and lens redshift $z$ are both unknown for 1 lens systems, only $z_{s}$ unknown for 4 lens systems and only $z$ unknown for 1 system.

\subsection{Basic Preparations}

The CLASS statistical sample use the flux density ratio $q_{r}$ of the multiple lensing images as a selection criterion of a sample: $q_{r}=\left|\mu_{+} / \mu_{-}\right| \leq 10$ (Chae et al.2002; Chen 2003a, 2003b), where the $\mu_{+}$and $\mu_{-}$are the magnifications of two (outside) images, respectively. The magnification of a image is determined by $\mu=\left[\frac{y}{x} \frac{d y}{d x}\right]^{-1}$. The parameter $q_{r}$ reduces the lensing cross section $\sigma$. For a NFW case, the influence of $q_{r}$ on $\sigma$ is very small and can be neglected. For an SIS lens, it needs to multiply the $\sigma$ by a factor $(9 / 11)^{2}$.

As the exact redshift distribution of the CLASS statistical sample is unknown, Chae et al.(2002) utilized a Gaussian model with mean redshift $\left\langle z_{s}\right\rangle=1.27$ given by Marlow et al. (2000) to describe the redshift distribution for the unlensed sources of the CLASS statistical sample. Denote this Gaussian model as $g^{c}\left(z_{s}\right)$, its distribution was explicitly plotted in Figure 5 of Chae (2003), which was obtained from describing the redshift distribution of the flat-spectrum sources as shown in that Figure 5. Note that as such an Gaussian model has a physical cut at the point $z_{s}=0$, it is no longer to have the standard form. Unlike the Gaussian model $g^{c}\left(z_{s}\right)$ by Chae (2003), here we shall take an alternative Gaussian model by directly fitting the redshift distribution of the subsample of CLASS statistical sample given by Marlow et al. (2000) instead of fitting the redshift distribution of the flat-spectrum sources in Chae (2003). Taking the general form of Gaussian model

$$
g\left(z_{s}\right)=\frac{N_{s}}{\sqrt{2 \pi} \lambda} \exp \left[\frac{-\left(z_{s}-a\right)^{2}}{2 \lambda^{2}}\right]
$$

with $N_{s}$ being the normalization parameter $\int_{0}^{\infty} f(x) d x \equiv 1$, and requiring the mean value $\int_{0}^{\infty} x f(x) d x \equiv 1.27$ given by Marlow et al. (2000), we then only need to fit the remaining 
one parameter. The best fit results are found to be

$$
N_{s}=1.6125 ; a=0.4224 ; \lambda=1.3761
$$

For comparison, we also present some results based on two treating methods appearing in literatures by using the CLASS statistical sample: the redshift distributions of sources are the average redshift value $d\left(z_{s}\right)=\delta\left(z_{s}-1.27\right.$ ) (see, e.g., Li \& Ostriker 2001) and $f\left(z_{s}\right)=0.204+0.2979 z_{s}-0.1121 z_{s}^{2}+0.001584 z_{s}^{3}$ (see, e.g., Sarbu et al. 2001). Fig. 6 gives curves of four models as a function of $z_{s}$ and the histogram of 27 CLASS subsample from Marlow et al. (2000).

Before comparing with the CLASS statistical sample, we should consider the effect of magnification bias $B$, which causes the overrepresentation of the lensed objects in a fluxlimited survey. The flux distribution of the CLASS statistical sample is well-described by $N(f) \propto\left(f / f_{0}\right)^{\eta}$ with $\eta=2.07 \pm 0.02(1.97 \pm 0.14)$ for $f \geq f_{0}\left(f \leq f_{0}\right)$ and $f_{0}=30 \mathrm{mJy}$ (Chae et al.2002). The analysis process of magnification bias is determined by the lensing equation and following equations

$$
\begin{aligned}
B & =\frac{\int_{A_{m}}^{\infty} \frac{d A}{A} p(A) \int_{f_{0}}^{\infty} N(f / A) d f}{\int_{f_{0}}^{\infty} d f N(f)} \\
A & =\Sigma A_{i} \\
& =\Sigma \frac{x_{i} d x}{y d y}=\Sigma \frac{x_{i} / y}{d y /\left.d x\right|_{i}} \\
p(A) & =\frac{p(y)}{|d A / d y|} ; p(y)=\alpha y ; \quad \int_{0}^{y_{\max }} p(y) d y=1,
\end{aligned}
$$

where $x$ and $y$ are the position parameters on the lens plane, $A$ is the total amplification of the multiply-imaged sources, $A_{m}$ is the minimum value of $A$ and $p(A)$ is the probability density for the amplification $A, y_{\max }$ is the maximum value of a source' position on the lens plane. $\alpha$ is the normalization constant of probability function $p(y)$ : for the SIS case $y_{\max }=1, \alpha=2$ and for the NFW case, it can be calculated out numerically by using $d y /\left.d x\right|_{y_{\max }}=0$. Then for the SIS case, after considering the influence of flux density ratio $q_{r}$, one needs only multiply the lensing probability by a constant factor 3.36. For the NFW case, B can be obtained from the numerical calculation. $P_{\text {obs }}(>\Delta \theta)$ and $d P_{\text {obs }}(>\Delta \theta) / d \Delta \theta$ are related to $P$ by an integration

$$
p(w) \equiv P_{\mathrm{obs}}(>\Delta \theta)=\iint B \frac{d P(>\Delta \theta)}{d z} \varphi\left(z_{s}\right) d z d z_{s},
$$

and

$$
q(w) \equiv \frac{d P_{\mathrm{obs}}(>\Delta \theta)}{d \Delta \theta}=\iint B \frac{d^{2} P(>\Delta \theta)}{d \Delta \theta d z} \varphi\left(z_{s}\right) d z d z_{s}
$$


with $\varphi\left(z_{s}\right)$ is the redshift distribution of sources.

We shall compare the theoretical results of the SIS case and NFW case with the CLASS statistical sample. Fig. 7 shows the lensing probability $P(>\Delta \theta)$ as a function of the splitting angle $\Delta \theta$ for the source redshift distribution $d\left(z_{s}\right), f\left(z_{s}\right), g^{c}\left(z_{s}\right)$ and $g\left(z_{s}\right)$. The thickest line in each panel is induced from the 13 observed lensing data. It is seen that the lensing probability $P(>\Delta \theta)$ of SIS case and NFW case are both sensitive to the parameter $w$, especially for NFW case. When parameter $w$ increases, the values of $P(>\Delta \theta)$ for both SIS and NFW cases clearly increase in the whole concerned ragion of $\Delta \theta$. Thus it is feasible to constrain the parameter $w$ from the SGL splitting angle data. The figure shows that the SIS model can only reproduce the data curve at small $\Delta \theta<1.5^{\prime \prime}$. When consider the rapid decline of $P(>\Delta \theta)$ from the data line at large $\Delta \theta$, a combined mechanism of SIS and NFW model is needed to explain the whole experimental curve. Define a new model parameter $M_{c}$ as Li \& Ostriker (2001): lenses with mass $M<M_{c}$ have the SIS profile, while lenses with mass $M>M_{c}$ have the NFW profile. Then the differential probability

$$
d P / d M=d P_{S I S} / d M \vartheta\left(M_{c}-M\right)+d P_{N F W} / d M \vartheta\left(M-M_{c}\right)
$$

where $\vartheta$ is the step function, $\vartheta(x-y)=1$, if $x>y$ and 0 otherwise. Because the splitting angle $\Delta \theta$ is directly proportional to the mass $M$ of lens halos, the contribution to large $\Delta \theta$ of SIS profile is depressed by $M_{c}$. The lens data require a mass threshold $M_{c} \sim 10^{13} h^{-1} M_{\odot}$, which is consistent with the halo mass whose cooling time equals the age of the universe today. In this note we shall use such a two-model combined mechanism to calculate lensing probabilities.

In Fig. 7, One can also find the influences of different source distributions on the lensing probability $P(>\Delta \theta)$ : the patterns of the function curves are hardly changed, but the function values for the same $\Delta \theta$ slightly increase from $d\left(z_{s}\right)$ to $f\left(z_{s}\right)$.

\subsection{Constraint on $w$}

We now come to our main purpose i.e., utilizing the SGL splitting angle statistic data from strong gravitational lenses to constrain the equation-of-state parameter $w$ of dark energy. For that, we define the likelihood function as

$$
\mathrm{L}(w)=(1-p(w))^{N-N_{l}} \prod_{i=1}^{N_{l}} q_{i}(w) .
$$

$p(w)$ represents the model-predicted lensing probabilities $P\left(>0.3^{\prime \prime}\right)$ of a source with the redshift distribution $\varphi\left(z_{s}\right)$ and can be calculated by using equations (23) , (33)), and (39). 
Here $\Delta \theta \geq 0.3^{\prime \prime}$ is an observational selection criteria. $q_{i}(w)$ is the model-predicted differential lensing probabilities $d P\left(>\Delta \theta_{i}\right) / d \Delta \theta_{i}$ of $i^{t h}$ observed lens system with the splitting angle $\Delta \theta_{i}$ and can be calculated by using equations (24), (34), and (40). To utilize the data informations adequately, we multiply the equation (39) by $\delta\left(z-z^{i}\right)$ or/and $\delta\left(z_{s}-z_{s}^{i}\right)$ (replace $\left.\varphi\left(z_{s}\right)\right)$ for the $i^{t h}$ observed lens system whose lens redshift $z^{i}$ or/and source redshift $z_{s}^{i}$ is/are known. For the unknown $z^{i}$ or $z_{s}^{i}$, we just integrate it out. Compared with utilizing the curve of lensing probability $P(>\Delta \theta)$ as a function of the splitting angle $\Delta \theta$ in the last subsection, which is introduced from lensed signals only, by using the likelihood function equation (41), the unlensed signals are utilized and their influences are quite important due to the large exponential number $\left(N-N_{L}\right)=8945$.

Firstly we discuss the possible constraints on the model parameter $M_{c}$ and the constant $w$. Under the given cosmological parameters $\left(\Omega_{M}, h, \sigma_{8}\right)=(0.24,0.73,0.74)$, Fig. 8 shows the $68 \%$ C.L. and $95 \%$ C.L. allowed regions from the CLASS statistical sample for the source redshift distribution $g\left(z_{s}\right), g^{c}\left(z_{s}\right), d\left(z_{s}\right)$ and $f\left(z_{s}\right)$, respectively. The crosshairs in three panels mark the best-fit points $\left(w, M_{c}\right)=(-0.89,1.37),(-0.94,1.36),(-1.4,1.68)$ and $(-0.73,1.27)$ from left to right, where the unit of $M_{c}$ is $10^{13} h^{-1} M_{\odot}$. The source redshift distributions $g\left(z_{s}\right)$ and $g^{c}\left(z_{s}\right)$ give nice constraints, while $d\left(z_{s}\right)$, which is not proper redshift distributions, provides somewhat strange unexpected results. The redshift distribution $f\left(z_{s}\right)$, which has a larger part of galaxies at high redshift, prefers a larger $w$ and smaller $M_{c}$. From now on we will only consider the $g\left(z_{s}\right)$ case and $g^{c}\left(z_{s}\right)$ case, which are extracted from the subsample of the CLASS statistical sample (Marlow et al. 2000). Our best fit result of $M_{c} \approx 1.40$ for both $g\left(z_{s}\right)$ case and $g^{c}\left(z_{s}\right)$ case is larger than the value $M_{c} \approx 1.0$ obtained by Li \& Ostriker 2002. The 95\% C.L. allowed regions of parameter $w$ for $g\left(z_{s}\right)$ case and $g^{c}\left(z_{s}\right)$ case are from -0.18 to -1.45 and from -0.11 to -1.85 , which are consistent with the $\Lambda$ CDM cosmology. The Fig.2 in Chae (2007) shows a much negative result of the parameter $w$. Our consideration here is based on the specially selected cosmological parameters, and also we have used the two-model combined mechanism, namely the utilization of model parameter $M_{c}$. As a consequence, our results avoid the large absolute value of parameter $w$.

In Fig. 9, we show the constraint for the parameters $\left(w_{0}, w_{a}\right)$ appearing in a time-varying equation-of-state $w(z)=w_{0}+w_{a} z /(1+z)$ under the given cosmological parameters $\left(\Omega_{M}, h\right)=$ $(0.24,0.73)$ for both $g\left(z_{s}\right)$ and $g^{c}\left(z_{s}\right)$ cases, and with two different values of $\sigma_{8}=0.74$ and $\sigma_{8}=0.90$. The crosshairs mark the best-fit points $\left(M_{c} ; w_{0}, w_{a}\right)=(1.36 ;-0.92,-1.31)$ for $g\left(z_{s}\right)$ case and $\left(M_{c} ; w_{0}, w_{a}\right)=(1.38 ;-0.89,-1.21)$ for $g^{c}\left(z_{s}\right)$ case when $\sigma_{8}=0.74$, and $\left(M_{c} ; w_{0}, w_{a}\right)=(1.56 ;-0.81,-2.5)$ for $g\left(z_{s}\right)$ case and $\left(M_{c} ; w_{0}, w_{a}\right)=(1.54 ;-0.83,-2.22)$ for $g^{c}\left(z_{s}\right)$ case when $\sigma_{8}=0.90$. The parameter $\sigma_{8}$ has significant influences on the mass power spectrum and the number density of dark halos, and our results show that the best fit $\left(w_{0}, w_{a}\right)$ are changed for the different selections of the parameter $\sigma_{8}$ : when $\sigma_{8}$ increases 
from $\sigma_{8}=0.74$ to $\sigma_{8}=0.9$, the best fit parameters $w_{0}$ increase moderately and the best fit parameters $w_{a}$ have a sizable decrease for both the $g\left(z_{s}\right)$ and the $g^{c}\left(z_{s}\right)$ cases.

After marginalizing the cosmological parameters $\left(\Omega_{M}, h, \sigma_{8}\right)$ and the critical mass parameters $M_{c}$ by Monte Carlo method, we obtain the constraint on $\left(w_{0}, w_{a}\right)$ in Fig. 10. For the three cosmological parameters, we assume the Gaussian prior distributions induced from the results of WMAP Three Year Data: $\left(\Omega_{M} \pm \sigma_{m}, h \pm \sigma_{h}, \sigma_{8} \pm \sigma_{\sigma_{8}}\right)=(0.238 \pm$ $0.019,0.73 \pm 0.03,0.74 \pm 0.06)$. For the model parameter $M_{c}$, we integrate it from 1.0 to 4.0. The crosshairs mark the best-fit point $\left(w_{0}, w_{a}\right)=(-0.88,-1.55)$ for $g\left(z_{s}\right)$ case and $\left(w_{0}, w_{a}\right)=(-0.91,-1.60)$ for $g^{c}\left(z_{s}\right)$ case. At the $95 \%$ C.L., our fitting results are consistent with that of Barger et al. (2006), and the SGL splitting angle statistic with the source redshift distribution $g\left(z_{s}\right)$ and $g^{c}\left(z_{s}\right)$ gives somewhat more negative values for the parameter $w_{a}$.

\section{CONCLUSIONS}

From the above analyzes, we have shown how the SGL splitting angle statistic can be used to quantitatively constrain the equation-of-state parameter $w$ of dark energy. Though due to the limited space-time, the difference of the parameter $w$ has few influences on the splitting angle $\Delta \theta$ and lensing cross section $\sigma$, while through the comoving number density of dark halos as sources and lenses described by Press-Schechter theory, dark energy can affect the efficiency with which dark-matter concentrations produce strong lensing signals. With a two model combined mechanism of dark halo density profile, which introduces a model parameter $M_{c}$, we have carefully investigated the constraints on constant $w$ and time-varying $w(z)=w_{0}+w_{a} z /(1+z)$. We find the best fit value $M_{c} \approx 1.4$ for both $w$ and $w(z)$, such a value is larger than the value $M_{c} \approx 1$ obtained by Li \& Ostriker 2002. This is mainly because in our analyzes both the lensed and unlensed signals have been utilized in the likelihood function equation (41), while in the analyzes by Li \& Ostriker 2002, only the lensed signals were be used. The transition from SIS to NFW characterized by the parameter $M_{c}$ is also motivated by the process of baryonic cooling (e.g., Kochanek \& White 2001), where the parameter $M_{c}$ was introduced to divide the cooled(SIS) and uncooled(NFW) halos. The estimated value by Kochanek \& White (2001) for $h=0.67$ is $M_{c} \approx 1 \times 10^{13} M_{\text {sun }}$ for the model without a bulge, which is also smaller than our fitting result. There are several differences between our calculations and theirs. Firstly, we use a larger Hubble constant $h=0.73>h=0.67$ and in our Figure 9, we show that for a larger h, we have a larger fit $M_{c}$. Secondly, the SIS model used by us has a larger relative lensing cross section than the exponential disk used by Kochanek \& White (2001), which gives a larger $M_{c}$. Thirdly, the ratio of the cluster to the 
background density $\Delta_{v i r}=\rho_{\text {halo }} / \rho_{M}>150$ used by us is much larger than the value $\Delta \approx 100$ used by Kochanek \& White (2001), which could also have some influences on the results. Nevertheless, all the fitting results for $M_{c}$ are consistent with appropriate considerations.

With the given cosmological parameters $\left(\Omega_{M}, h, \sigma_{8}\right)=(0.24,0.73,0.74)$, we have compared the results of constant $w$ corresponding to four kinds of source redshift distributions. It has been shown that $d\left(z_{s}\right)$ is not suitable for the SGL data analysis. For the redshift distributions of normalized Gaussian-type model $g\left(z_{s}\right)$ and Gaussian model $g^{c}\left(z_{s}\right)$, the fitting results are $\left(w, M_{c}\right)=\left(-0.89_{-0.26}^{+0.49}, 1.37_{-0.33}^{+0.47}\right)$ and $\left(w, M_{c}\right)=\left(-0.94_{-0.16}^{+0.57}, 1.36_{-0.34}^{+0.47}\right)$ respectively, and the fitting results for the constant $w$ are consistent with the $\Lambda \mathrm{CDM}$ at $95 \%$ C.L.. For the time-varying $w(z)$, we have firstly investigated the influence of $\sigma_{8}$ with the redshift distributions $g\left(z_{s}\right)$ and $g^{c}\left(z_{s}\right)$ and found that the fitting results of the double parameters $\left(w_{0}, w_{a}\right)$ are changed when $\sigma_{8}$ increases from $\sigma_{8}=0.74$ to $\sigma_{8}=0.9$. With the above given cosmological parameters, the best fitting results for the $g\left(z_{s}\right)$ case are $\left(M_{c} ; w_{0}, w_{a}\right)=(1.36 ;-0.92,-1.31)$ for $\sigma_{8}=0.74$ and $\left(M_{c} ; w_{0}, w_{a}\right)=(1.56 ;-0.81,-2.5)$ for $\sigma_{8}=0.9 ;$ and for $g^{c}\left(z_{s}\right)$ case, the best fit results are $\left(M_{c} ; w_{0}, w_{a}\right)=(1.38 ;-0.89,-1.21)$ for $\sigma_{8}=0.74$ and $\left(M_{c} ; w_{0}, w_{a}\right)=(1.54 ;-0.83,-2.22)$ for $\sigma_{8}=0.9$.

After marginalizing our likelihood functions over the cosmological parameters $\left(\Omega_{M}, h, \sigma_{8}\right)$ (by using the prior probabilities induced from the WMAP Three Year data) and the model parameter $M_{c}$, we have obtained a reliable constraint on the parameters $\left(w_{0}, w_{a}\right)$. Within the allowed uncertainties, the results for $w_{0}$ are consistent with the constraints obtained from the Type Ia supernovae data (Barger, Guarnaccia \& Marfatia 2006). Our fit results are $\left(w_{0}, w_{a}\right)=\left(-0.88_{-1.03}^{+0.65},-1.55_{-1.88}^{+1.77}\right)$ for $g\left(z_{s}\right)$ case and $\left(w_{0}, w_{a}\right)=\left(-0.91_{-1.46}^{+0.60},-1.60_{-2.57}^{+1.60}\right)$ for $g^{c}\left(z_{s}\right)$ case. It is noticed that the best fitting results based on the SGL splitting angle statistic favor negative values for the parameter $w_{a}$, which differs from the best fitting values obtained based on the Type Ia supernovae data, where the best fitting results favor positive values for the parameter $w_{a}$ (Barger, Guarnaccia \& Marfatia 2006). A combining constraint is interesting and will be investigated elsewhere.

In conclusion, the quantitative investigation has shown that the SGL splitting angle statistic can lead to a consistent constraint on the constant $w$ and the double parameters $\left(w_{0}, w_{a}\right)$ of the time-varying dark energy equation of state $w(z)=w_{0}+w_{a} z /(1+z)$. Especially for the allowed range of parameters $\left(w_{0}, w_{a}\right)$, the SGL splitting angle statistic does give an interesting bound. It can be seen from Fig.8 to Fig.10 that the normalized Gaussian-type source redshift distribution $g\left(z_{s}\right)$ leads to the most stringent constraints. Though it does not yet allow to obtain a more accurate constraint, while it can provide a complementarity to other constraints from Supernovae, cosmic microwave background, weak lensing. 


\section{Acknowledgments}

The authors would like to thank R.G. Cai, X.M. Zhang, and Z.H. Zhu for useful discussions. The authors also acknowledge the useful comments from referee. This work was supported in part by the National Science Foundation of China (NSFC) under the grant \# 10821504,10475105, 10491306 and the Project of Knowledge Innovation Program (PKIP) of Chinese Academy of Science. 


\section{REFERENCES}

Barger, V. Guarnaccia, E \& Marfatia, D. 2006, Phys.Lett. B635 61-65

Basilakos, S. \& Plionis, M. 2006, ApJ 650 L1

Browne, IW.A., et al. 2003, MNRAS, 341, 13

Bullock, J. S., Kolatt, T. S., Sigad, Y., et al. 2001, MNRAS, 321, 559

Caldwell, R. R, Dave, R., \& Steinhardt P. J. 1998, Phys. Rev. Lett., 80, 1582

Chae, K.-H. et al. 2002, Phys. Rev. Lett., 89, 15

Chae, K.-H. et al. 2003, MNRAS, 346,746C

Chae, K.-H. 2007, ApJ, 658, 71

Chen, D. -M. 2003a, A\&A, 397, 415

Chen, D. -M. 2003b, ApJ, 587, L55

Eisenstein, D. J., \& Hu, W. 1999, ApJ, 511, 5

Fedeli, C., \& Bartelmann, M. 2007, A\&A, 461, 49

Hanyu, C. \& Habe, A. 2001, ApJ, 554,1268

Henstock, D., Browne, I., Wilkinson, P., \& McMahon, R. 1997, MNRAS, 290, 380

Kochanek, C. S. \& White, M. 2001, ApJ, 559, 531

Kuhlen, M., Keeton, C. R. and Madau, P. 2004, ApJ, 601, 104

Li, L. -X., \& Ostriker, J.P. 2002, ApJ, 566, 652

Li, L. -X., \& Ostriker, J.P. 2003, ApJ, 595, 603

Linder, E. 2004, Phys.Rev. D, 70, 043534

Ma, C. P., Caldwell, R. R. \& Wang, L. M. 1999 ApJ, 521, 1

Marlow, D. R., Rusin, D., Jackson, N., Wilkinson, P. N., \& Browne, I. W. A. 2000, AJ, 119, 2629

Navarro, J. F., Frenk, C. S., \& White S. D., M. 1995, MNRAS, 275, 720 
Navarro, J. F., Frenk, C. S., \& White, S. D. M. 1996, ApJ, 462, 563

Navarro, J. F., Frenk, C. S., \& White, S. D. M. 1997, ApJ, 490, 493

Peebles P. J. E., \& Ratra, B. 2003, Rev. Mod. Phys. 75, 559

Phillips, P. M., et al. 2001, MNRAS, 328, 1001

Porciani, C., \& Madau, P. 2000, ApJ, 532, 679

Riess, A. G., et al. 1998b, AJ, 116, 1009

Riess, A. G., et al. 2004, ApJ, 607, 665

Rusin, D., \& Ma, C. -P. 2001, ApJ, 549, L33

Sarbu, N., Rusin, D., \& Ma, C.-P. 2001, ApJ, 561, L147

Schneider, P., Ehlers, J., \& Falco, E. E. 1992, Gravitational Lenses (Berlin: Springer-Verlag)

Smail, I., Hogg, D. W., Yan, L., \& Cohen, J. G. 1995, ApJ, 449, L105

Spergel, D. N., et al. 2003, ApJS, 148 , 175

Spergel, D. N., et al. 2007, ApJS, 170, 335

Wang, L. \& Steinhardt, P.J. 1998, ApJ, 508, 483

Weinberg, N. N., \& Kamionkowski, M. 2002, MNRAS, 000, 1

Zhao, H. S., Haehnelt, M. G., \& Rees, M. J. 2002, New Astronomy, 7, 385 


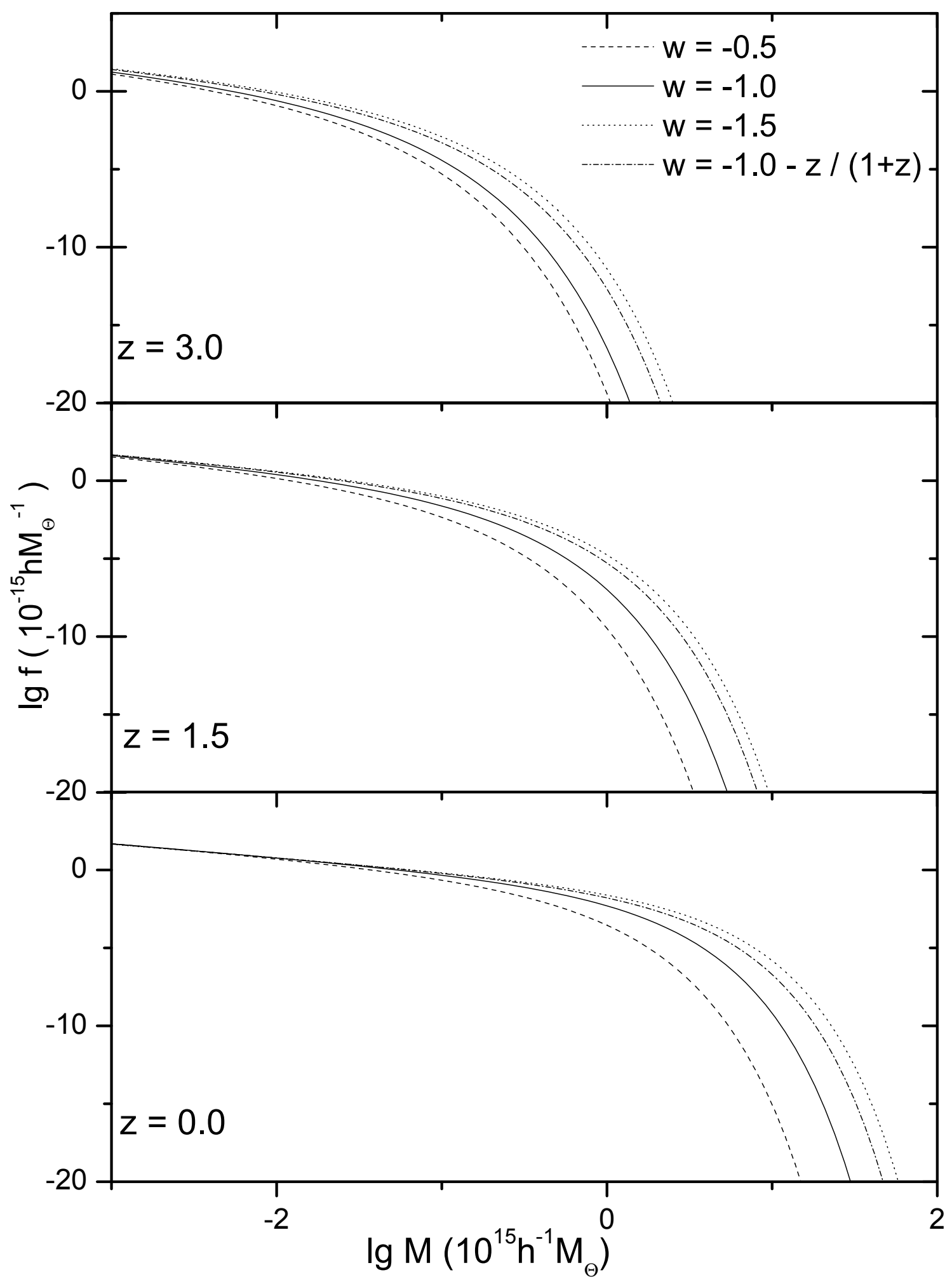




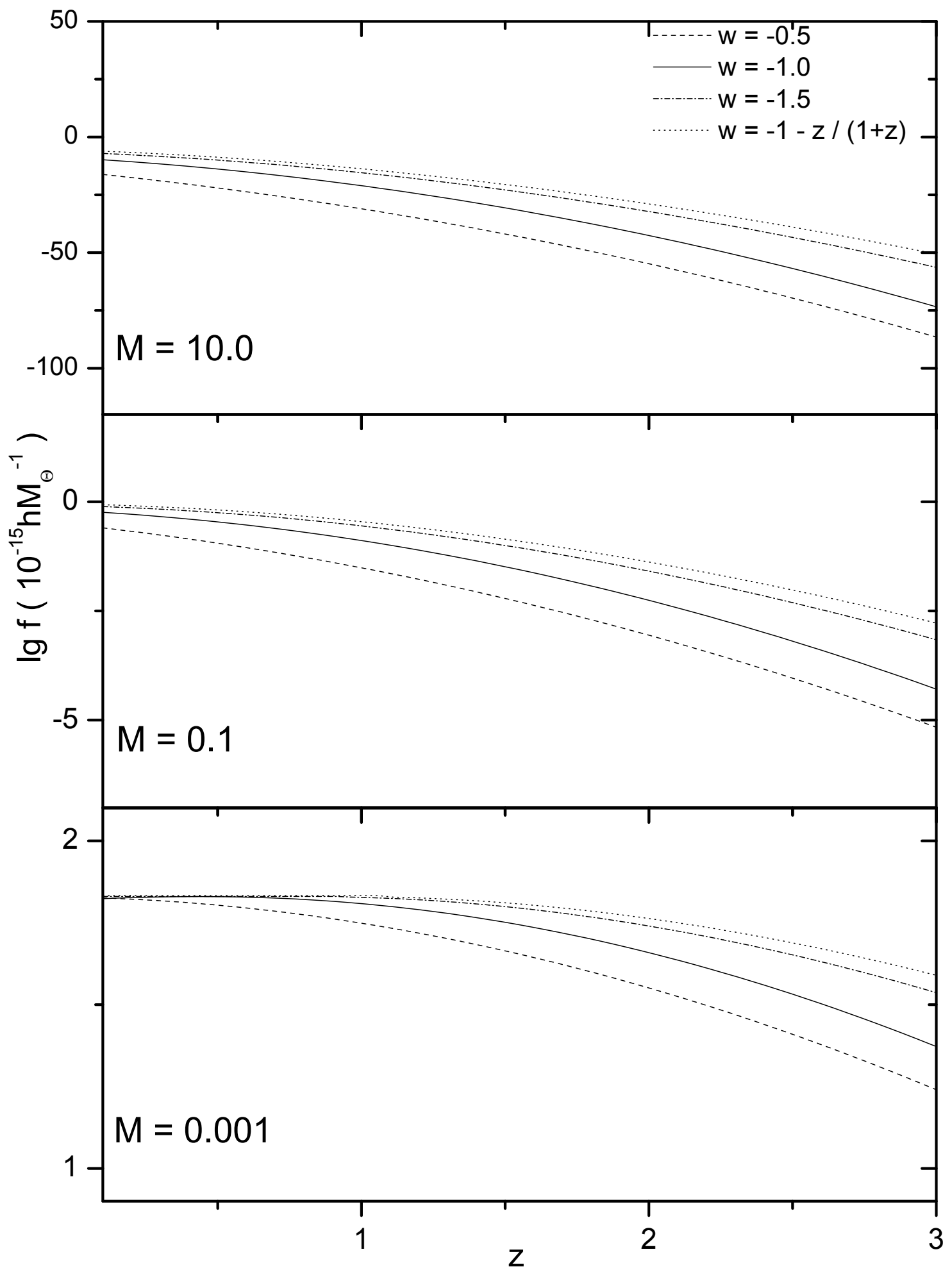




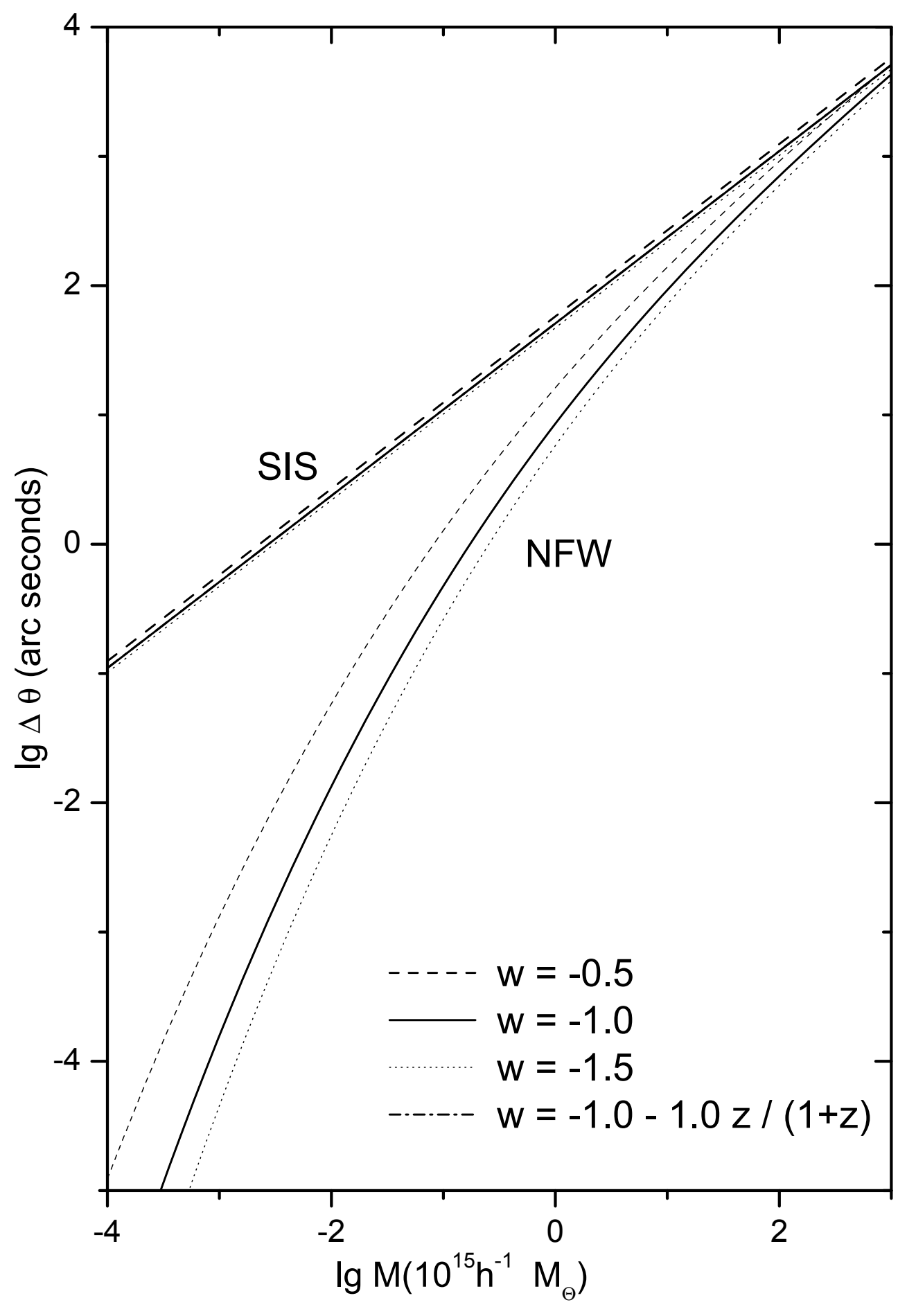




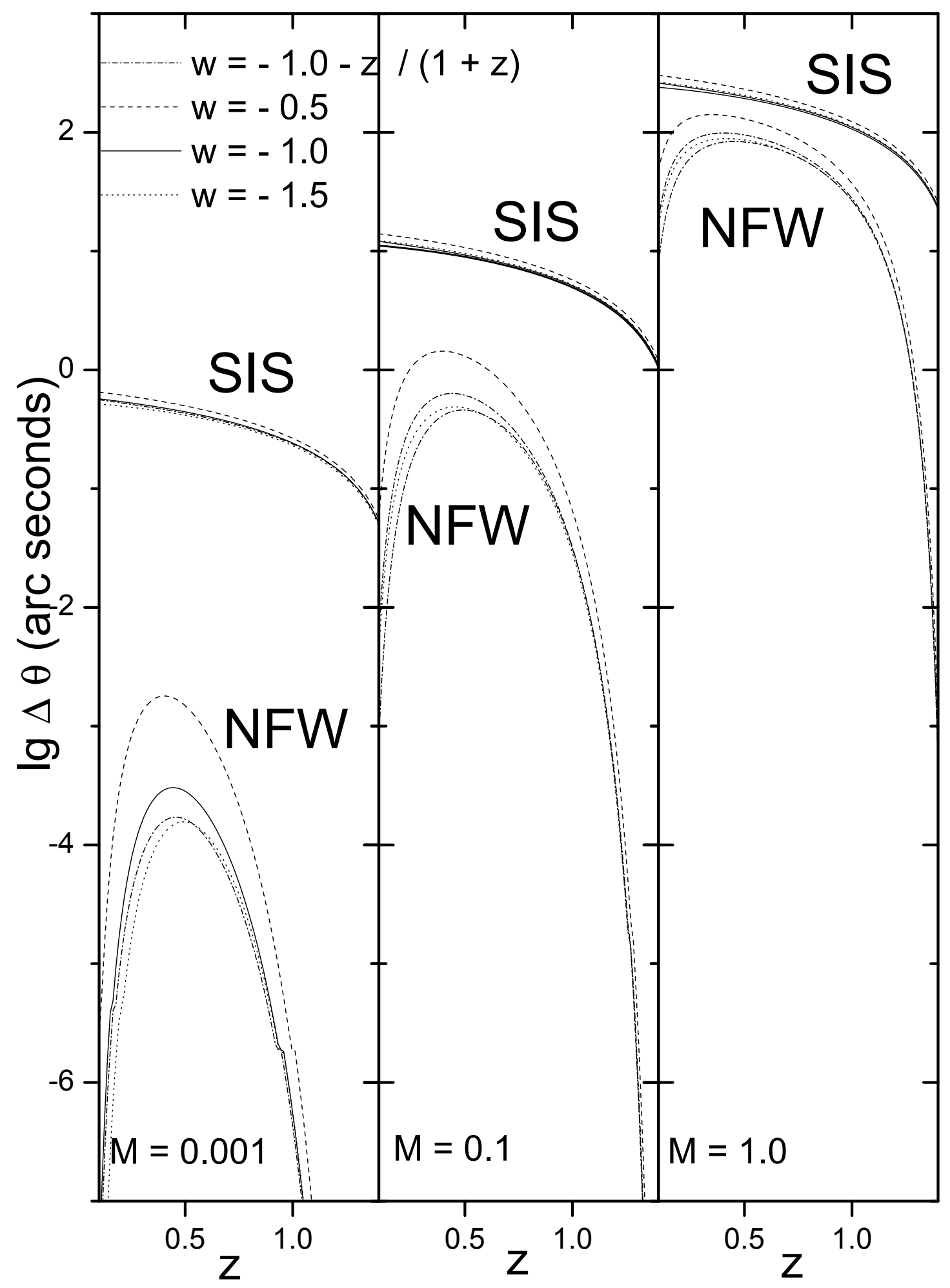




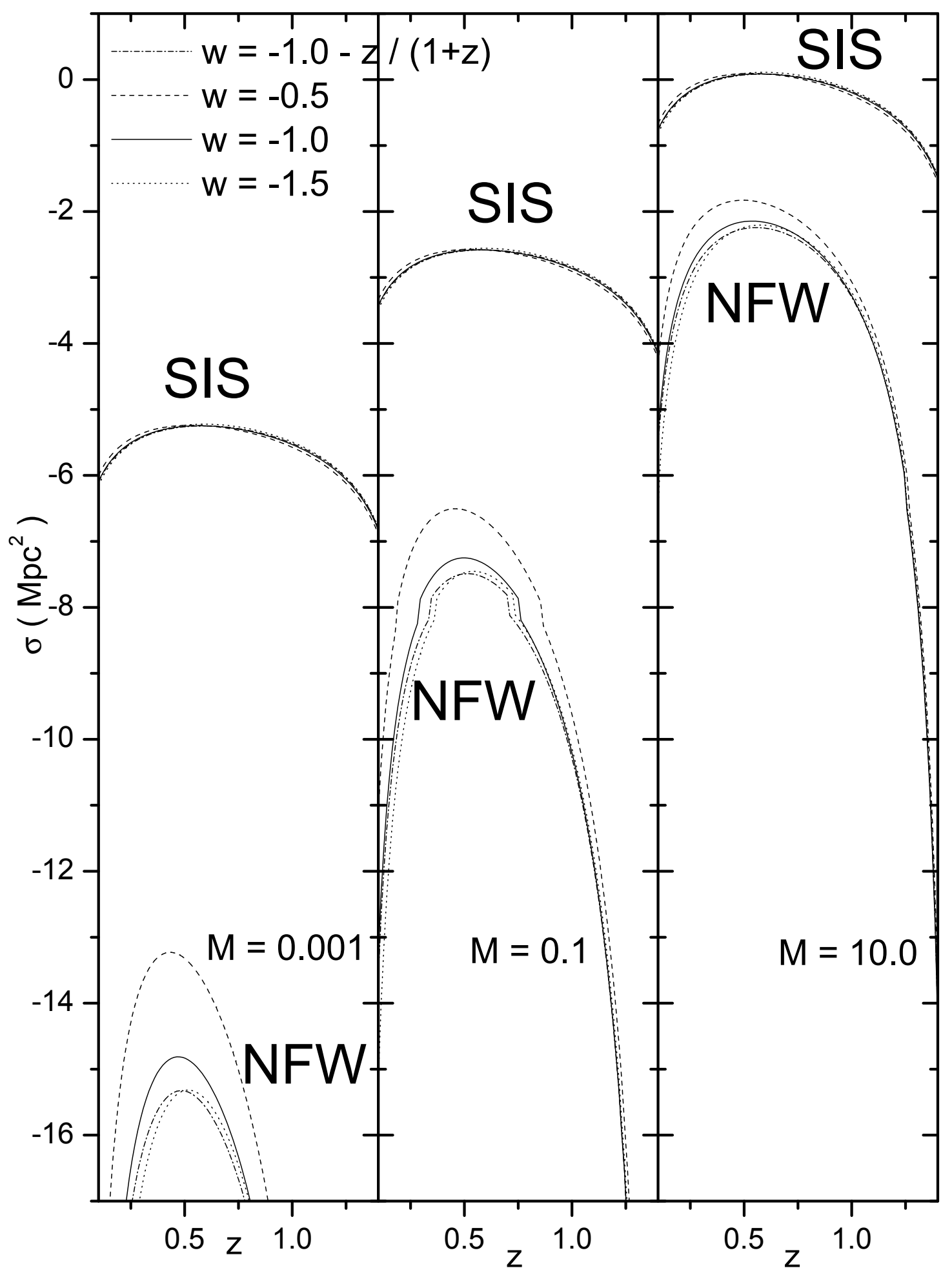




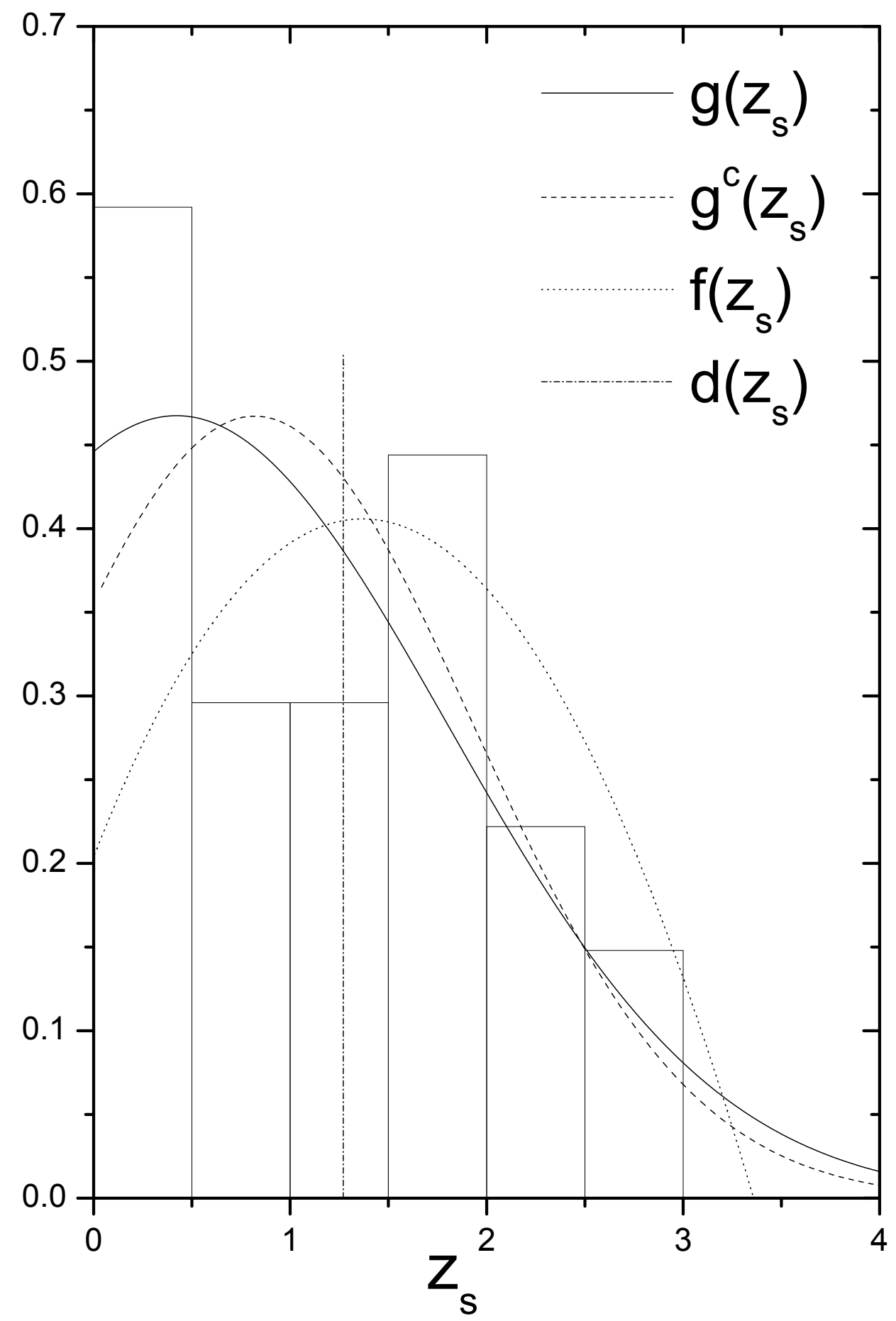




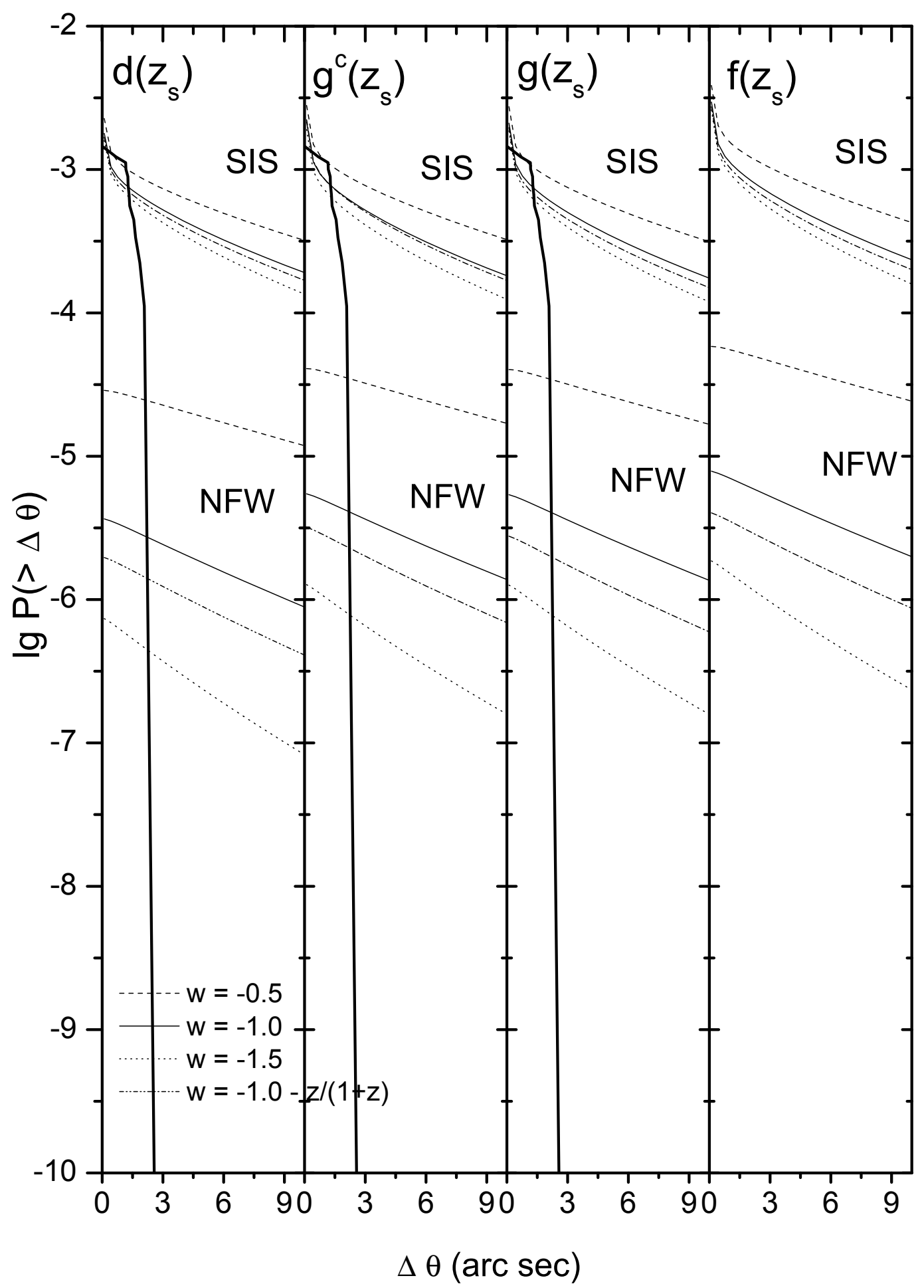




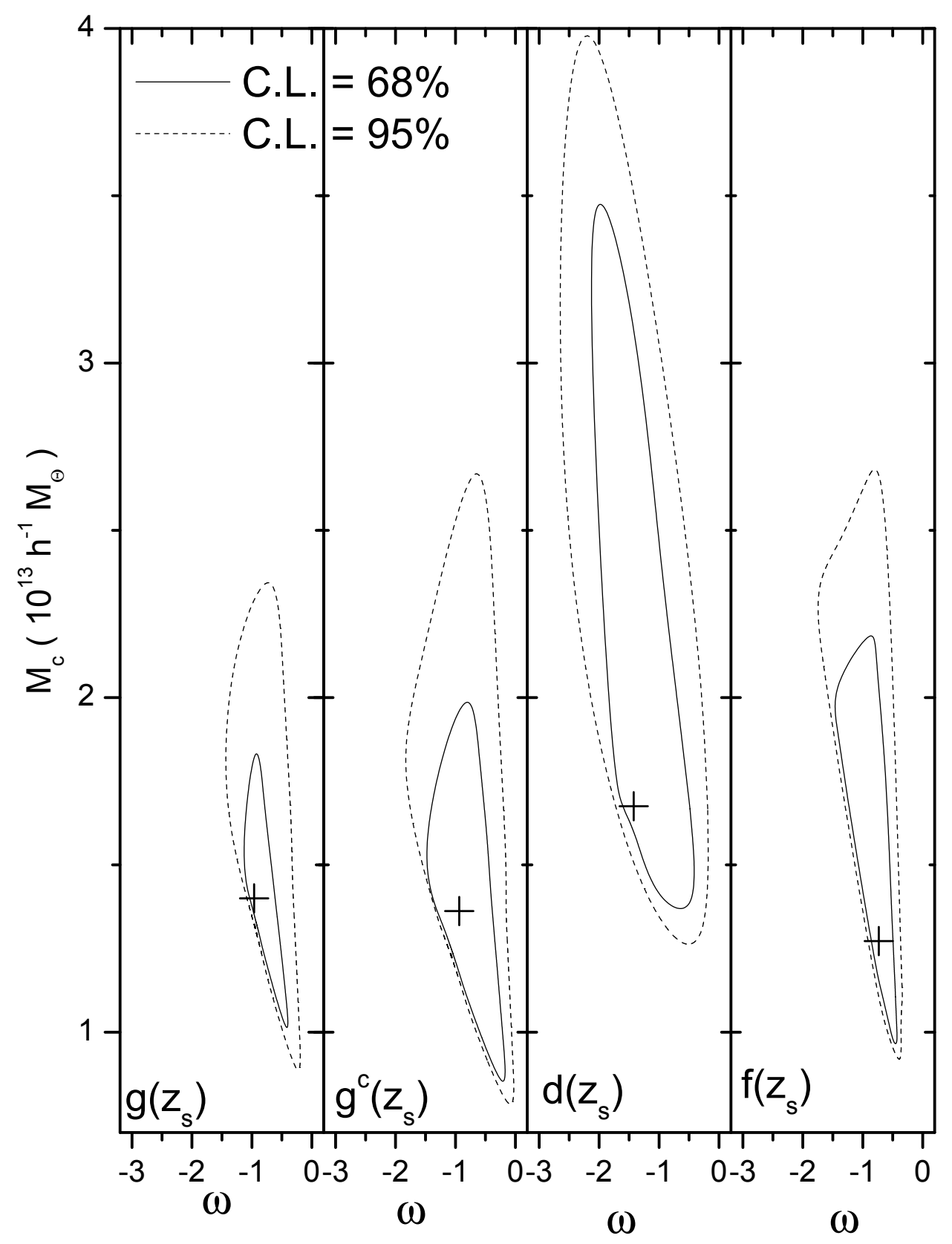

Fig. 8.-68\% C.L. and 95\% C.L. allowed regions from the CLASS statistical sample for the source redshift distribution $g\left(z_{s}\right), g^{c}\left(z_{s}\right), d\left(z_{s}\right)$ and $f\left(z_{s}\right)$, respectively. The crosshairs in three panels mark the best-fit points $\left(w, M_{c}\right)=(-0.89,1.37),(-0.94,1.36),(-1.4,1.68)$ and $(-0.73,1.27)$ from left to right. 


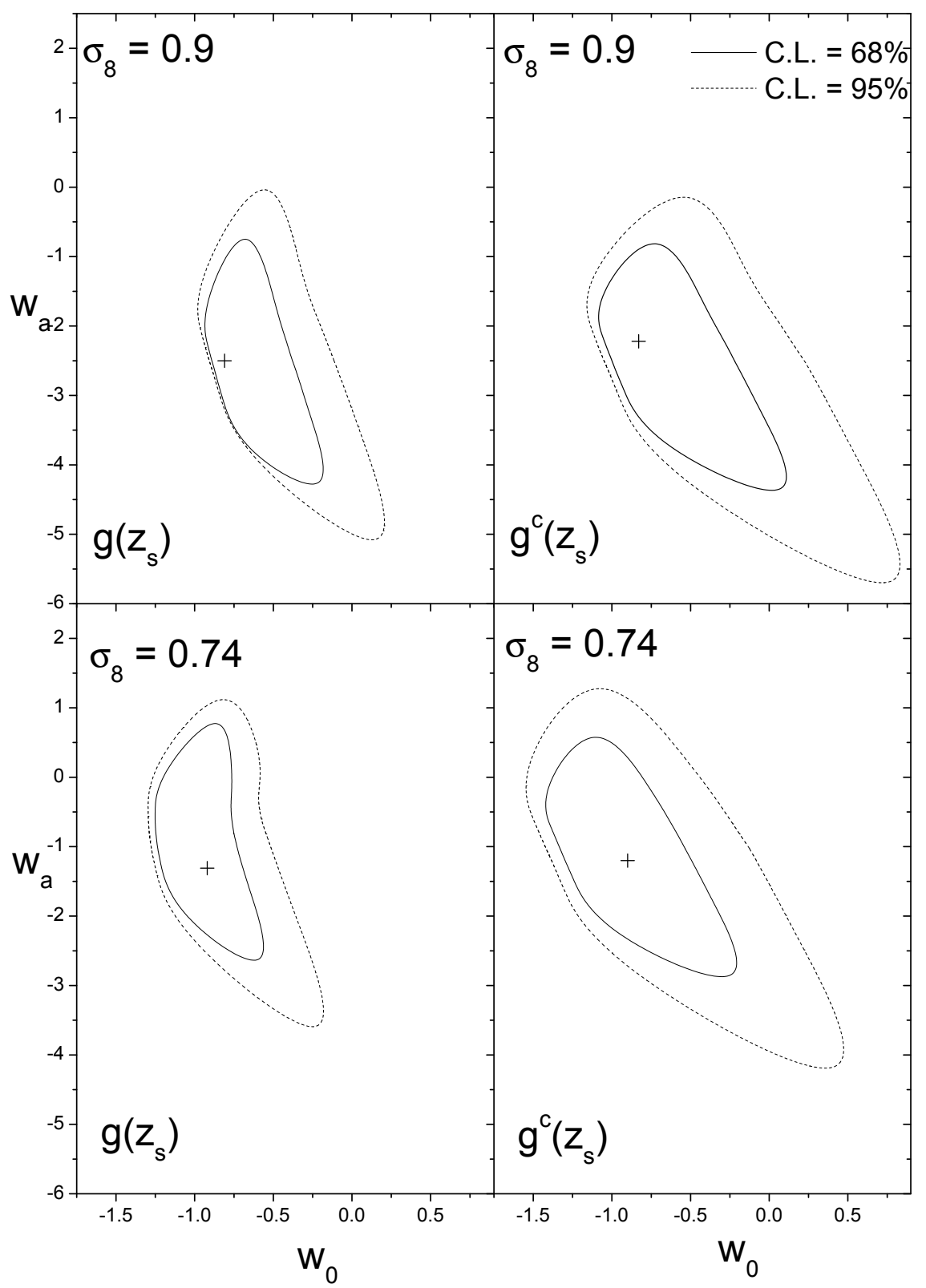

Fig. 9.- $68 \%$ C.L. and $95 \%$ C.L. allowed regions from the CLASS statistical sample for the $f\left(z_{s}\right)$ redshift distribution with different $\sigma_{8}=0.74$ and 0.9 , respectively. The crosshairs mark the best-fit points: for the $g\left(z_{s}\right)$ case are $\left(M_{c} ; w_{0}, w_{a}\right)=(1.36 ;-0.92,-1.31)$ for $\sigma_{8}=0.74$ and $\left(M_{c} ; w_{0}, w_{a}\right)=(1.56 ;-0.81,-2.5)$ for $\sigma_{8}=0.9$; and for $g^{c}\left(z_{s}\right)$ case, the best fit results are $\left(M_{c} ; w_{0}, w_{a}\right)=(1.38 ;-0.89,-1.21)$ for $\sigma_{8}=0.74$ and $\left(M_{c} ; w_{0}, w_{a}\right)=(1.54 ;-0.83,-2.22)$ for $\sigma_{8}=0.9$ 


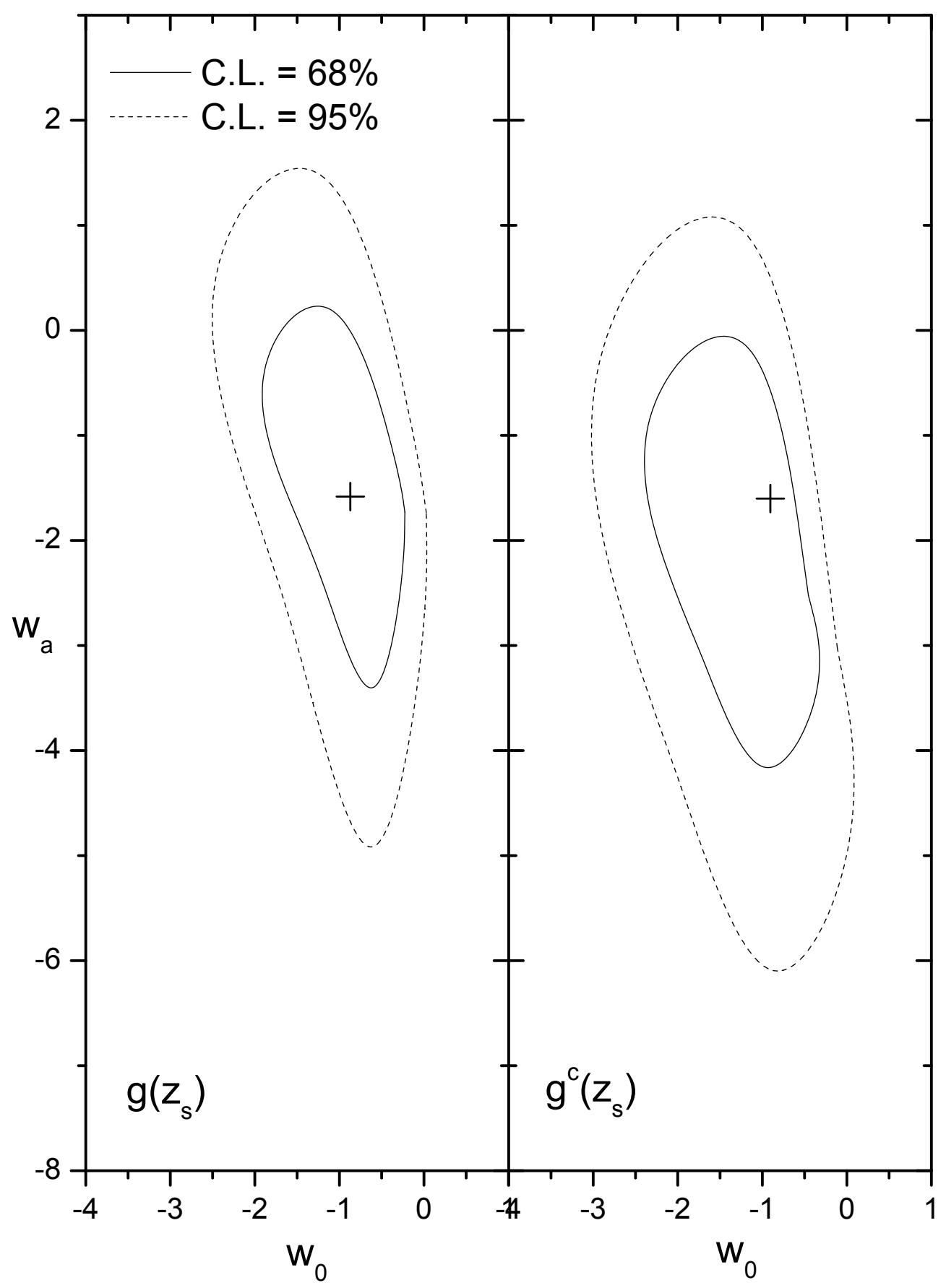

Fig. 10.-68\% C.L. and 95\% C.L. allowed regions from the CLASS statistical sample for the source redshift distribution $f\left(z_{s}\right)$. The crosshirs mark the best-fit points $\left(w_{0}, w_{a}\right)=(-0.88,-1.55)$ for $g\left(z_{s}\right)$ case and $\left(w_{0}, w_{a}\right)=(-0.91,-1.60)$ for $g^{c}\left(z_{s}\right)$ case . The cosmological parameters $\left(\Omega_{M}, h, \sigma_{8}\right)$ and the model parameter $M_{c}$ have been marginalized 